\title{
A WUSCHEL-like homeobox gene, OsWOX3B responses to NUDA/GL-1 locus in rice
}

\author{
Honglei Zhang ${ }^{1 \dagger}$, Kun Wu ${ }^{2+}$, Yufeng Wang ${ }^{1}$, Yu Peng ${ }^{1}$, Fengyi Hu ${ }^{3}$, Lu Wen ${ }^{4}$, Bin Han ${ }^{1}$, Qian Qian ${ }^{2 *}$ \\ and Sheng Teng ${ }^{1 *}$
}

\begin{abstract}
Background: Most of the rice varieties are pubescent. However, the presence of trichomes is an undesirable characteristic in rice production because trichomes can cause atmospheric pollution. The use of glabrous rice varieties represents a solution to this problem. Yunnan Nuda Rice, a glabrous cultivar that constitutes approximately 20\% of rice germplasms in Yunnan can provide important recourse for breeding of glabrous rice varieties.

Results: The "Nuda" phenotype in Yunnan Nuda Rice was found to be controlled by a single recessive allelic gene within the well-characterized GL-1 locus. A high-resolution genetic and physical map was constructed using 1,192 Nuda individuals from the $F_{2}$ population that was delivered from the cross between the Yunnan Nuda variety HMK and the pubescent TN1 variety. The NUDA/GL-1 gene was mapped to a $28.5 \mathrm{~kb}$ region containing six annotated genes based on the Nipponbare genomic sequence. By comparing the sequences and expression patterns of different pubescent and glabrous varieties, LOC_Os05902730, a WUSCHEL-like homeobox gene (OsWOX3B) was identified as the candidate gene. This hypothesis was confirmed by RNA interference (RNAi) and transgenic complementation. Trichome deficiency in RNAi lines was associated with increased efficiency of grain packaging but did not affect the main agronomic traits.
\end{abstract}

Conclusion: NUDA/GL-1 locus encodes OsWOX3B gene.

Keywords: Yunnan nuda rice, Nuda/glabrous, Map-based cloning, WUSCHEL-like homeobox gene OsWOX3B

\section{Background}

Trichomes are ubiquitous in land plants (Southwood 1986; Werker 2000). The density, morphology, and chemical composition of leaf hairs vary widely and these factors contribute to their diverse physiological, physical and chemical functions (Southwood 1986; Werker 2000). However, the presence of trichomes is an undesirable characteristic in rice production due to the generation of dust during harvesting and grain manipulating processes. Trichomes are important causes of atmospheric pollution and these pollutants can cause acute and chronic irritation of the eyes, skin, and respiratory tract, which subsequently result in intolerable itching

\footnotetext{
* Correspondence: qianqian188@hotmail.com; steng@sibs.ac.cn

${ }^{\dagger}$ Equal contributors

'Shanghai Institute of Plant Physiology and Ecology, Shanghai Institute for Biological Sciences, The Chinese Academy of Sciences, Shanghai 200032,

China

${ }^{2}$ State Key Laboratory of Rice Biology, China National Rice Research Institute, Chinese Academy of Agricultural Sciences, Hangzhou 310006, China Full list of author information is available at the end of the article
}

and allergy, and even longstanding diseases such as Rice Miller's Syndrome (Lim et al. 1984). Therefore, the use of glabrous rice varieties, which are trichome-deficient on the leaves and glumes, represents a solution to this problem.

Natural and artificial glabrous mutants have been identified in model plants such as Arabidopsis (Karkkainen and Agren 2002) and cereal crop species including rice (Foster and Rutger 1978), maize (Moose et al. 2004), wheat (Leisle 1974) barley (Sato and Takeda 1992) oats (Sarkarung and Collins 1977) pearl millet (Kumar and Andrews 1993) and sorghum (Gibson and Maiti 1983). In particular, trichome formation in Arabidopsis has been used as a model system to study various developmental and cellular mechanisms in plants (Hulskamp 2004; Marks 1997). With mutagenesis screens, dozens of genes involved in trichome initiation, spacing, and shape have been identified in Arabidopsis (Marks 1997; Hulskamp and Schnittger 1998 Serna and Martin 2006 Szymanski et al. 2000). These genes encode several classes 
of transcriptional factors, including MYB (GL1) (Larkin et al. 1993; Oppenheimer et al. 1991), WD-40 (TTG1) (Walker et al. 1999), bHLH (GL3/EGL3) (Payne et al. 2000) and HD-ZIP (GL2) (Johnson et al. 2002; Szymanski et al. 2000). Recent studies of Arabidopsis have shown a complex that consists of GL1-GL3/EGL3-TTG1 promote GL2 expression to regulate hair cell differentiation (Payne et al. 2000; Lloyd et al. 2000). Several other MYB proteins, including CYC, TRY (Schellmann et al. 2002) and ETC1 (Kirik et al. 2004), compete with GL1 by interacting with bHLH proteins to repress trichome initiation on the leaf (Schellmann et al. 2002). However, no glabrous genes have been cloned in cereal crop species to date.

Glabrous rice varieties have been identified in different locations worldwide including America, Yunnan in China and regions of south-eastern and southern Asia. Two loci (GL-1 and GL-2) for glabrous leaf and hull characteristics have been identified by classic genetic analysis. Two complementary genes ( $\mathrm{Hla}$ and $\mathrm{Hlb}$ ) have been identified for long pubescence on leaves, and one gene $(\mathrm{Hg})$ has been identified for long pubescence on floral hulls (Nagao et al. 1960). Among these genes, only the GL-1 gene from American glabrous rice has been mapped to chromosome 5 with the use of restriction fragment length polymorphism (RFLP) markers (Yu et al. 1995). This locus was further narrowed to a region of approximately $157 \mathrm{~kb}$ (Wang et al. 2009) and $54 \mathrm{~kb}$ (Li et al. 2010) by two independent research groups.

Yunnan is a center of rice landrace diversity. It is reported that about 20\% of the Yunnan landraces are glabrous rice varieties, known as Yunnan Nuda Rice (Qin et al. 1997). In this study, the glabrous phenotypes of Yunnan upland Nuda rice landrace HMK were characterized. By genetic analysis, the glabrous phenotype of HMK was shown to be conferred by a pair of recessive genes, and this locus was allelic with the reported GL-1 locus. Furthermore, the NUDA/GL1 gene was cloned using a map-based approach and identified as a WUSCHEL-like gene (LOC_Os05g02730) belonging to the homeodomain (HD) subfamily.

\section{Results}

\section{Characterization of Yunnan nuda rice HMK variety}

The presence of trichomes is ubiquitous in rice varieties. Leaf and grain phenotypes of the pubescent TN1 variety and the glabrous Yunnan Nuda Rice HMK variety were examined using stereomicroscopy and scanning electron microscopy (SEM).

Trichomes were observed on the upper (adaxial) surface and along the veins of the leaf blade and on glumes of pubescent rice varieties, such as TN1 (Figures 1a, 1b). Three types of trichomes were observed under SEM of the leaf: macro, micro and glandular hairs (Figure 1e). Macro hairs were located on silica cells over a thin vascular bundle, whereas micro and glandular hairs were located along the stomata cells or adjacent to motor cells (Figure 1f). Very few macro hairs were observed on the lower (abaxial) surface of the leaf (Figure 1g). On the glumes, hairs were distributed on the valleys between two swelled longitudinal veins (Figure 1h).

Both the leaf and hull are glabrous and smooth in Yunnan Nuda Rice variety HMK (Figures 1c and 1d). Small bulges were present in place of macro hairs on the leaves (Figure 1i), although glandular hairs and occasional micro hairs were still observed (Figure 1j). There were no macro hairs on the lower (abaxial) surface
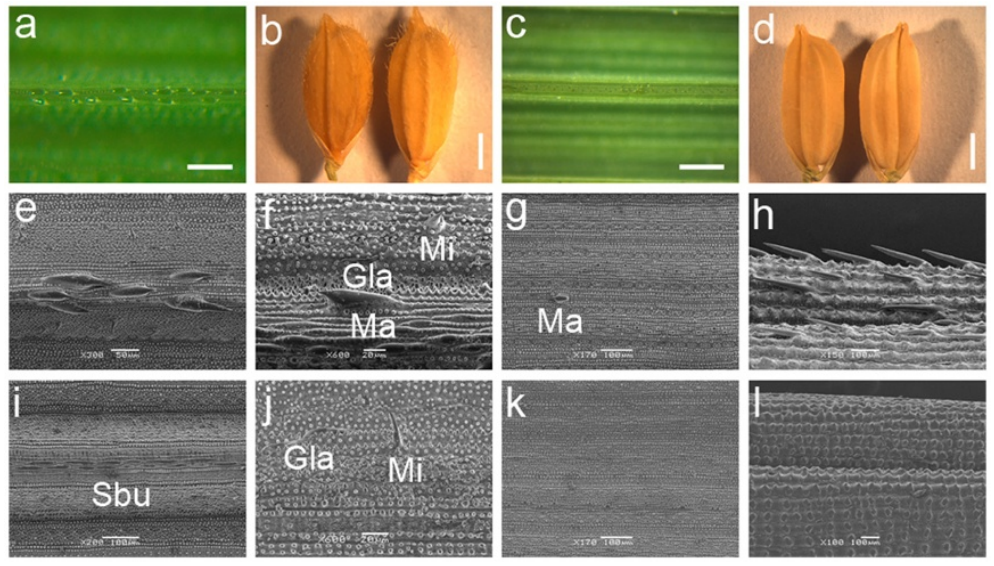

Figure 1 Leaf and grain phenotypes of pubescent variety TN1 and glabrous Yunnan Nuda rice variety, HMK. a TN1 leaf adaxial epidermis morphology (Scale bar: $0.5 \mathrm{~mm})$. b TN1 grain morphology (Scale bar: 1.5mm). c HMK leaf adaxial epidermis morphology (Scale bar: $0.5 \mathrm{~mm})$. d HMK grain morphology (Scale bar: 1.5mm). e, $\mathbf{f}$ Scanning electron microscope (SEM) images of TN1 leaf adaxial epidermis. $\mathbf{g}$ SEM images of TN1 leaf abaxial epidermis. $\mathbf{h}$ SEM images of TN1 grain epidermis. $\mathbf{i}$, j SEM images of HMK leaf adaxial epidermis. $\mathbf{k}$ SEM images of HMK leaf abaxial epidermis. I SEM images of HMK grain epidermis. Ma, macro hairs; Mi, micro hairs; Gla, glandular hairs; Sbu, small bulges. 
(Figure 1k). No hairs were observed on the surface of hulls (Figure 1l).

\section{Genetic analysis and fine mapping of the NUDA locus}

The genetic basis of the Nuda phenotype was analyzed. All $F_{1}$ hybrids resulting from the cross between TN1 and a Yunnan Nuda Rice variety, HMK, exhibited a trichomous leaf phenotype. One-hundred and fifty-eight $\mathrm{F}_{2}$ individuals were screened and 120 trichomous leaf individuals and 38 glabrous individuals were identified. A three to one $\left(\chi^{2}=0.076, \chi^{2} 0.05=3.84\right)$ segregation ratio was determined. These results indicated that the Nuda phenotype was controlled by a single pair of recessive genes.

American rice varieties commonly exhibit a glabrous phenotype similar to that of Yunnan Nuda Rice. The GL-1 gene conferring this phenotype has been mapped to the start of chromosome 5 (Chr. 5). To determine whether NUDA is allelic to GL-1, the HMK variety was crossed with the glabrous American variety, Lemont. All $F_{1}$ plants exhibited a glabrous phenotype (Figure $2 \mathrm{a}-\mathrm{c}$ ), which indicated that the NUDA locus is allelic to GL-1.

Simple sequence repeat (SSR) markers located at the start of Chr. 5 were used for the primary mapping of NUDA. Linkage analysis of $158 \mathrm{~F}_{2}$ individuals showed linkage of the RM17713 and RM13 markers with the NUDA locus at distances of 7.7 and $7.2 \mathrm{cM}$, respectively
(Figure 2d). The NUDA locus was mapped between markers GL2 and GL8 (Figure 2d). A high-resolution genetic and physical map for the fine mapping of NUDA was constructed by the analysis of 11 newly developed markers, including 10 insertion/deletion (InDel) markers and one single nucleotide polymorphism (SNP) marker in 1,192 $\mathrm{F}_{2}$ Nuda individuals derived from the TN1 and HMK cross (Table 1). The NUDA locus was mapped to a $28.5 \mathrm{~kb}$ interval between markers GL7 and GLSNP (Figure 2e). Six annotated genes were identified in this defined interval based in P1 artificial chromosome (PAC) clone AP001111 from the Nipponbare variety. These were LOC_Os05g02710 (retrotransposon protein), LOC_Os05g02720(hypothetical protein), LOC_Os05g02730 (WUSCHEL-related homeobox 3B), LOC_Os05g02740 (expressed protein), LOC_Os05g02750 (membrane protein) and LOC_Os05g02754 (expressed protein) (Figure 2f).

\section{NUDA/GL-1 candidate genes}

Genomic sequences of the candidate region defined by the markers GL7 and GLSNP were retrieved from the sequence of Nipponbare PAC clone P0699E04 (AP001111) and 9311 contig Ctg015203 (AAAA02015203). A large deletion including LOC_OsO5g02710 and LOC_Os05g02720 in 9311 was confirmed by PCR and sequence analyses, indicating that these genes do not encode NUDA/GL-1 in the pubescent Nipponbare and 9311 varieties (Figure 2g).
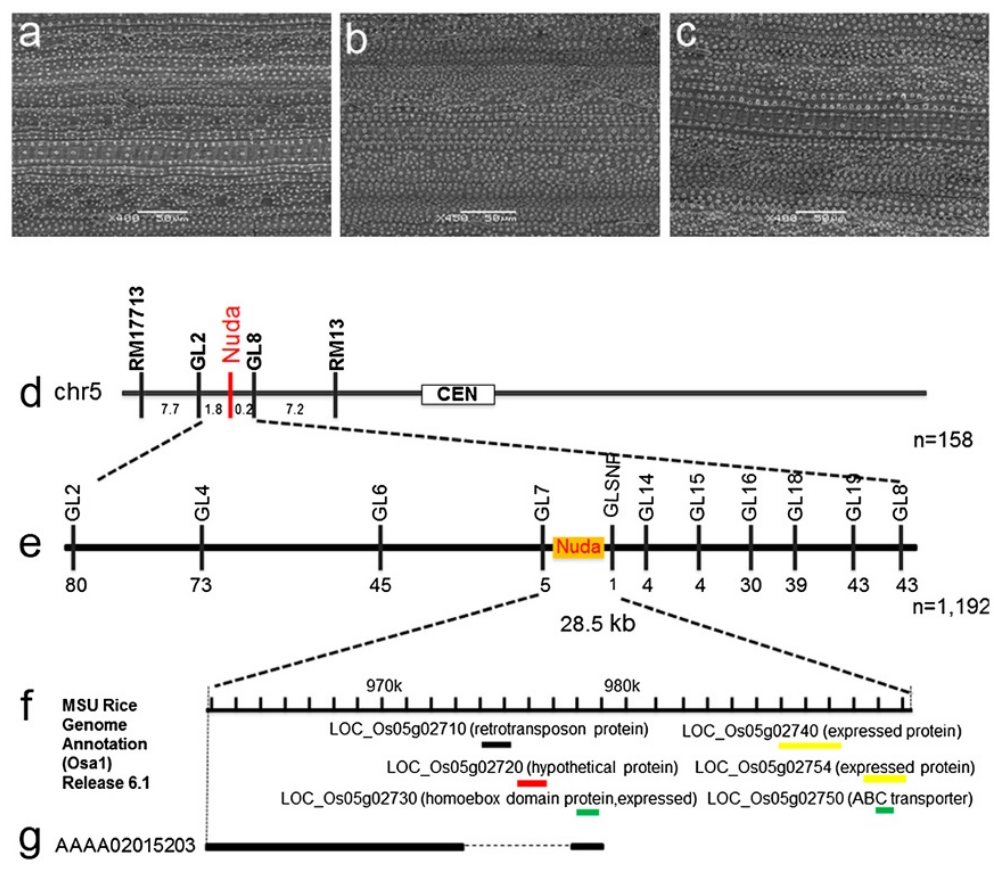

Figure 2 Genetic analysis and fine mapping of NUDA/GL-1. a SEM images of HMK leaf adaxial epidermis. $\mathbf{b}$ SEM images of Lemont leaf epidermis. c SEM images of HMK/Lemont $F_{1}$ leaf epidermis. $\mathbf{d}$ Location of NUDA on rice chromosome 5 in $158 F_{2}$ plants. Numbers under the linkage map indicate genetic distance between adjacent markers. e High-resolution linkage map of the NUDA region produced with $1,192 \mathrm{~F}_{2}$ plants. The number of recombinants between markers is indicated under the linkage map. $\mathbf{f}$ Six putative genes were annotated within the 28.5 kb region according to MSU Rice Genome Annotation Release 6.1. $\mathbf{g}$ The large deletion in 9311 (Dotted line). 
Table 1 Markers used for NUDA/GL-1 gene mapping

\begin{tabular}{|c|c|c|c|}
\hline Marker name & Forward primer( $\left(5^{\prime}-3^{\prime}\right)$ & Reverse primer(5'-3') & Marker type \\
\hline RM17713 & TTGTAACCACCAGCAGCAGGG & AGCAATGGTACAAATAGCCAAGC & SSR \\
\hline GL2 & AGTAAAATTGGATGTCATTTGGTA & CAAGAAAACTTCAGTACATTGGTG & InDel \\
\hline GL4 & CCGCTACGGAAGGGTAATAATG & AGTCGAAAGGATGGGAGGAGAA & InDel \\
\hline GL6 & TTATACTITCATACGCACGATG & AACCTAGCTGATGGGTCTAGAT & InDel \\
\hline GL7 & GGGTTTGGGTGGTCCTCTC & CATGCACGCCGAGTAGCT & InDel \\
\hline GLSNP & GCCGGTGATCGACAACGCCA & TGGGATCAGCTGAAAGTCTGTCCA & Direct sequence \\
\hline GL14 & GCCGTGCAACACGATATGG & GGCTTGTTTGGCGGTCAC & InDel \\
\hline GL15 & AGTGCCACAAATATAGCTCCG & CATITGATCTATTATAGTGATGATT & InDel \\
\hline GL16 & CACCGCTTTACGAACGCC & AGCAGCCACCTTCACGAG & InDel \\
\hline GL18 & TACCTGGGCGCATGAACA & CGGCAAGTIITTGGGCTA & InDel \\
\hline GL19 & TTTACTAGAAAAGATGCTCTGTT & CCACAATAAATTTCACATTAGC & InDel \\
\hline GL8 & TGTACATTCGATTACACGAGATAA & GCGAGAGGTGAGTAGGGCT & InDel \\
\hline RM13 & TCCAACATGGCAAGAGAGAG & TATCACATTCGATTCCAGCATG & SSR \\
\hline
\end{tabular}

The remaining four candidate genes were identified as follows: $L O C \_O s 05 g 02750$ encodes a bacterial-type ATP binding cassette $(\mathrm{ABC})$ transporter protein involved in efflux transport activity specific for UDPglucose and aluminum tolerance in rice ( $\mathrm{Ma}$ et al. 2009); LOC_Os05g02740 and LOC_Os05g02754 encode expressed proteins with unknown functions; and LOC_Os05g02730, a WUSCHEL-like homeobox gene (OsWOX3B).

The sequences and expression patterns of these four genes were investigated to identify the NUDA/GL-1 candidate gene. The sequences of these genes were identical to those of Nipponbare. Therefore, the expression patterns of these genes were further investigated in leaves and young panicles from HMK and TN1 varieties. A similar expression pattern of LOC_Os05g02740 and LOC_Os05g02750/ LOC_Os05g02754 was observed in both varieties. LOC_Os05g02730 expression was detected in the panicles of TN1 but not HMK, although expression of this gene was not detected at vegetative stage in either variety (Figure 3). These results implicated LOC_Os05g02730 (OsWOX3B) as the NUDA/GL1 candidate gene.

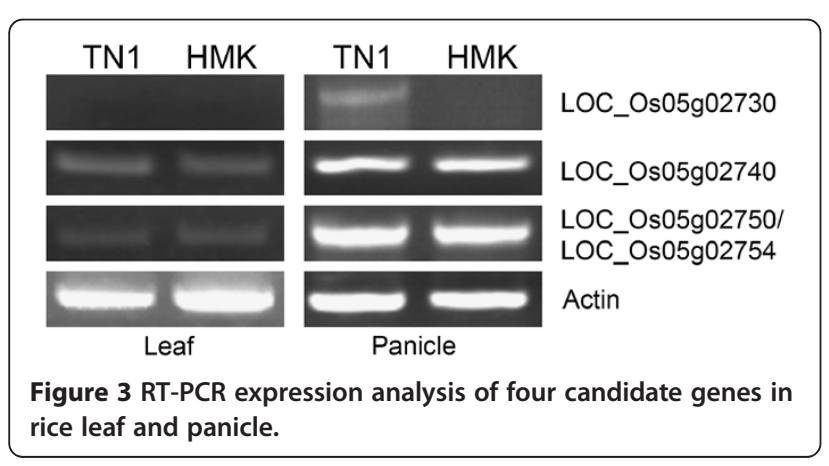

RNAi and genetic complementation of the NUDA/GL-1 gene

RNAi and genetic complementation analyses were performed to confirm that $O s W O X 3 B$ corresponded to $N U D A / G L-1$. The transfer of an RNAi construct designed to repress $O s W O X 3 B$ expression into Nipponbare resulted in transgenic lines that exhibited a glabrous phenotype in leaves (Figure 4a-d) and hulls (Figure $4 \mathrm{e}-\mathrm{h}$ ) from T0 generation. Furthermore, a $4.2 \mathrm{~kb}$ fragment including the promoter and coding region of OsWOX3B was cloned into pCAMBIA1301 and transferred into HMK. Pubescent transgenic rice plants with trichomes on the leaves were obtained, although the shapes of the trichomes were different and the alignments of the cells were disordered, which probably were due to the ectopic expression of OsWOX3B in transgenic plants (Figure 4i-k). These results indicated that the OsWOX3B corresponded to the NUDA/GL-1 gene and was responsible for the glabrous phenotype in HMK Nuda rice.

\section{NUDA/GL-1/OsWOX3B encodes a member of WOX3 subfamily}

Both the 5' and 3' ends of the NUDA/GL-1/OsWOX3B cDNA were obtained by the rapid amplification of CDNA ends (RACE) from the mRNA extracted from the panicles of the pubescent variety. The full length of NUDA/GL-1/OsWOX3B cDNA is $1019 \mathrm{bp}$, including an open reading frame with $780 \mathrm{bp}$, a 5'-untranslated region (UTR) with $132 \mathrm{bp}$ and a 3'-UTR with $107 \mathrm{bp}$ (Figure 5a). By comparing the sequences of the full length cDNA and the relevant genomic region, two introns were identified (Figure $5 \mathrm{~b}$ ). The putative protein contains a homeodomain (HD), which is a DNA-binding domain and a WUSCHEL domain, which indicated it 

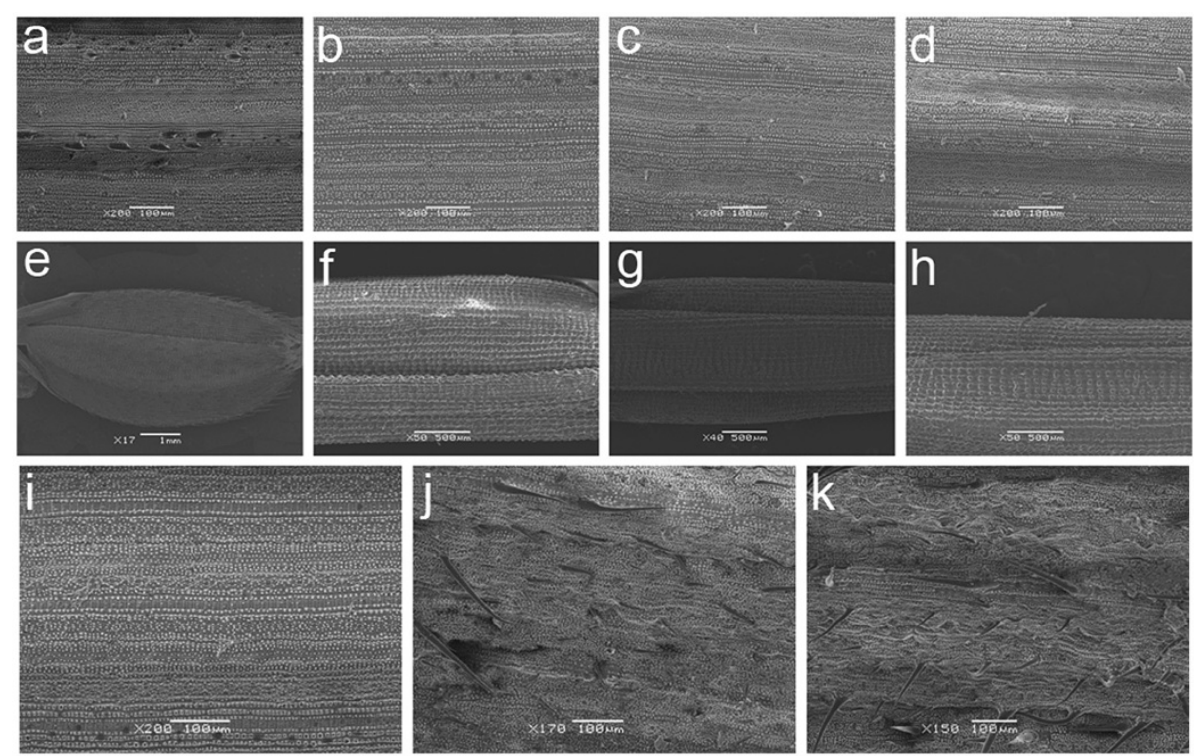

Figure 4 SEM images of adaxial epidermis of leaf and grain. a Nipponbare leaf b OsWOX3/NUDA RNAi line T1325 leaf, c T1326 leaf, d T1328 leaf, e Nipponbare grain, f OsWOX3/NUDA RNAi line T1325 grain, g T1326 grain, h T1328 grain, i leaf of HMK, j complementation transgenic line 1 leaf, $\mathbf{k}$ complementation transgenic line 2 leaf.

belonged to the WUSCHEL-like homeobox (WOX) gene family (Figure 5a).

Fourteen OsWOX genes and fifteen AtWOX genes were identified based on previous studies (Dai et al. 2007; Haecker et al. 2004 Nardmann et al. 2007 Vandenbussche et al. 2009 Zhang et al. 2010) and BLAST results in this study. BLAST searches of the NCBI Non-redundant Protein Sequences Database (http://blast.ncbi.nlm.nih.gov/) were performed using the homeodomain sequence of AtWUS (At2g17950) (Haecker et al. 2004) as a query. Phylogenetic analysis of these genes and four maize WOX genes, which belong to the same subgroup of $O s W O X 3$, was performed based on the sequences of the homeodomain regions. OsWOX3B was shown to be a homolog of AtWOX3/PRS (Figure 5c). In contrast to Arabidopsis, three rice genes and four maize genes were identified in the WOX3 subfamily. The phylogenetic tree map indicated that genes of WOX3 subfamily were divided into several subgroups. LOC_Os11g01130 (also known as OsNS2 or OsWOX3) (Zhang et al. 2010; Dai et al. 2007), LOC_Os12g01120 (also known as OsNS or OsNS1) (Nardmann et al. 2007; Zhang et al. 2010), $Z m N S 1$ and ZmNS2 were tightly related and close to AtWOX3/PRS. NUDA/GL-1/OsWOX3B was in a separate subgroup (Figure $5 \mathrm{c}$ ).

The expression patterns of NUDA/GL-1/OsWOX3B in the root, shoot apical meristem (SAM), leaf sheath, leaf blade and panicle from the TN1 and HMK varieties were detected by RT-PCR. The expression of this gene could be detected in the panicle but not in the root, SAM, sheath or blade in pubescent rice TN1. In Nuda Rice
HMK, no expression was detected in each tested tissue (Figure 6).

\section{Trichome deficiency increases efficiency of grain packaging}

The agronomic traits of Nipponbare and three OsWOX3B/NUDA/GL-1 RNAi transgenic lines were investigated. No significant differences were observed in terms of plant height, tiller number, specklet number per panicle, seed setting percentage and grain weight in RNAi lines compared with Nipponbare (Additional file 1: Table S1). These results indicated that OsWOX3B/ NUDA/GL-1 RNAi had no effect on these agronomic traits. Similarly, no significant differences were observed in grain size and shape, including grain length, grain width, grain thickness and ratio of length and width in RNAi lines compared with Nipponbare. These findings indicated that OsWOX3B/NUDA/GL-1 RNAi did not affect the size and shape of grains (Figure 7a, Additional file 2: Figure S1). However, the efficiency of grain packaging was increased in the three RNAi transgenic lines as measured by the number and weight of dried grains in a $100 \mathrm{ml}$ volume (Nipponbare, 2,119.2 \pm 23.0 grains weighing $49.6 \pm 0.5 \mathrm{~g}$; three OsWOX3B/NUDA/GL-1 RNAi transgenic lines: $2,432.7 \pm 18.4$ grains weighing $57.8 \pm 0.5 \mathrm{~g}$; $2,406.5 \pm 9.1$ grains weighing $57.6 \pm 0.2$ g; and 2,431.9 \pm 9.2 grains weighing $57.9 \pm 0.2 \mathrm{~g}$ ) (Figure $7 \mathrm{~b}$ ).

\section{Discussion}

The glabrous trait is beneficial for rice production due to the consequent reduction in dust pollution during grain 


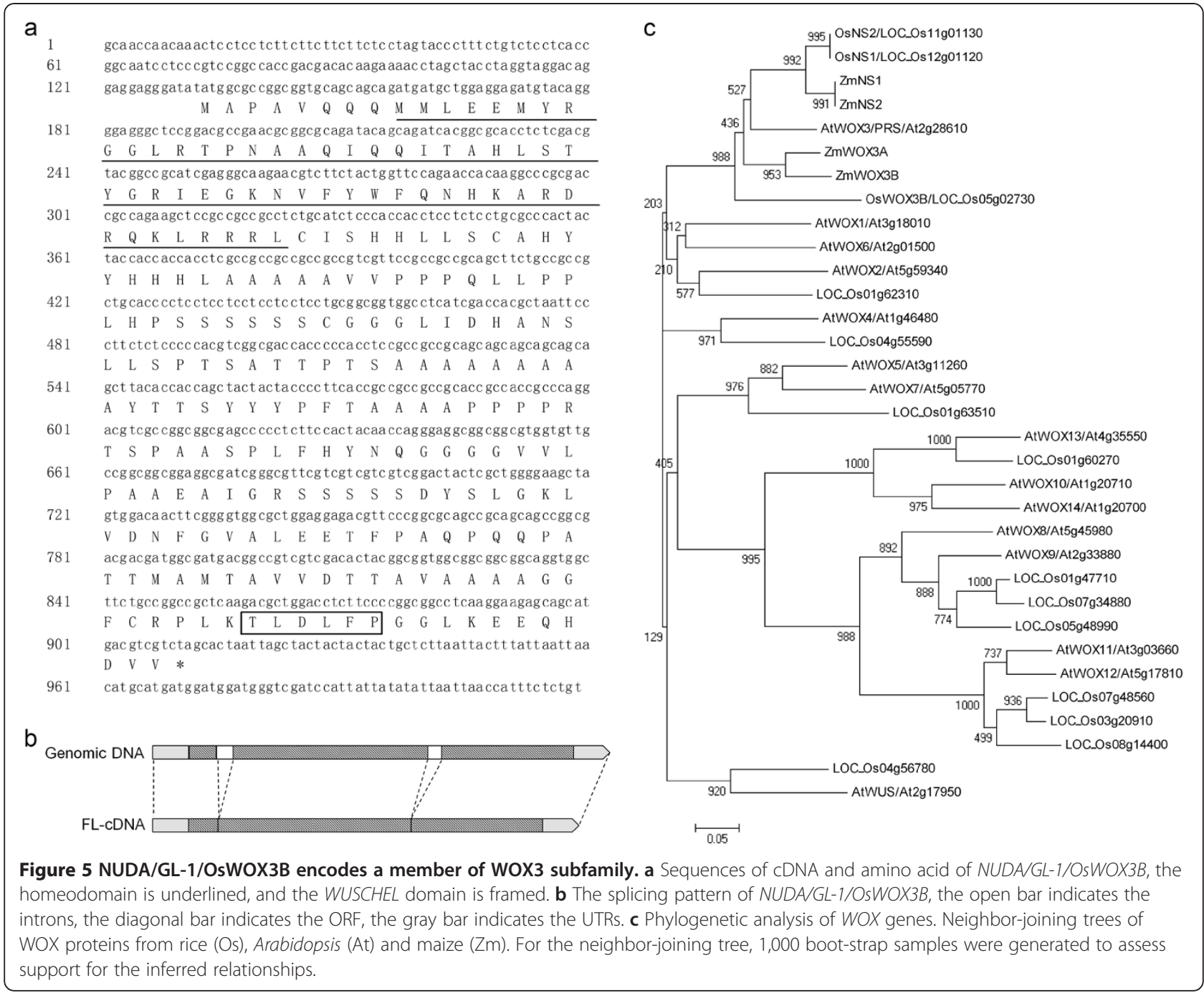

harvesting, drying and processing. Furthermore, it has been observed that the bulk density of the glabrous rice varieties was higher (Rutger and Mackill 2001). Theoretically, the lack of trichomes can make the glabrous kernels packed closer because the spaces occupied by the trichomes are released. It is clear that the size and shape of the grains also affect the grain packaging efficiency. In this study, the grain size and shape of OsWOX3B RNAi lines were similar with those of wild type plants. These

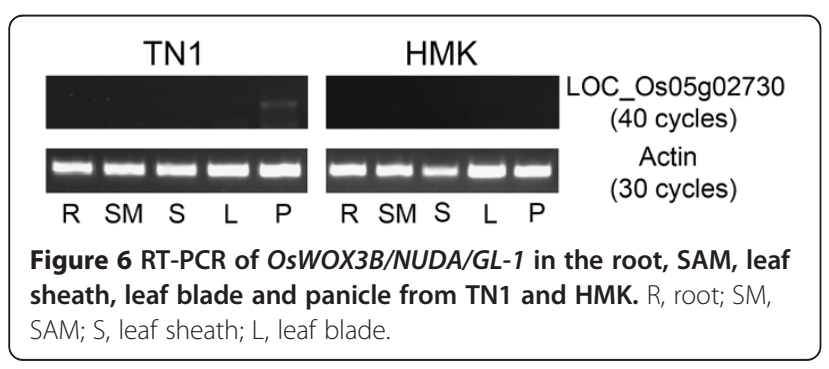

strictly isogenic materials provided convincing evidence that lack of glum hair truly increased the efficiency of grain packaging. Glabrous rice varieties have been found in many locations worldwide and glabrous germplasm has been used to develop glabrous varieties. Studies suggest the existence of at least two loci that confer the glabrous phenotype in leaves and hulls although they have not been cloned. In this study, the NUDA locus of Yunnan Nuda Rice was demonstrated to be allelic to the $G L$ 1 locus of American glabrous varieties. Furthermore, the NUDA/GL-1 gene was cloned by using a map-based approach and the locus was shown to encode a WUSCHEL-like homeobox gene (OsWOX3B) by RNAi and complementation transgenic studies.

Trichome is an excellent model system to study cell fate determination, cell cycle regulation, cell polarity and cell expansion in the model plant species, Arabidopsis. Recently, a number of genes that control the initiation and morphogenesis of trichomes have been identified 

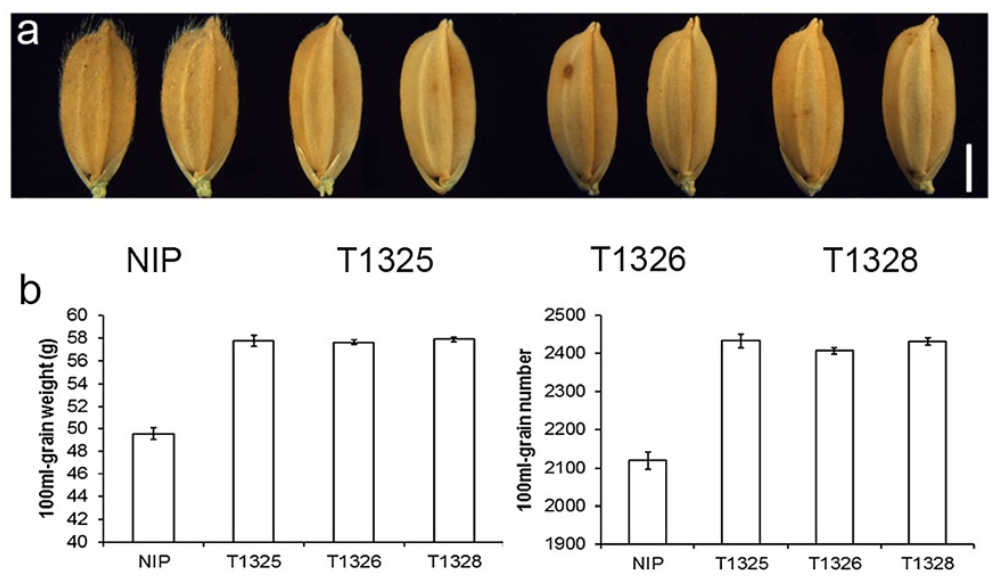

Figure 7 Trichome deficiency increases efficiency of grain packaging. a Grains of Nippare and three NUDA/GL-1/OsWOX3B RNAi transgenic lines (Scale bar: $2 \mathrm{~mm}$ ). b Packed dry grain numbers and weights of Nipponbare and three OsWOX3B/NUDA/GL1 RNAi transgenic lines in $100 \mathrm{ml}$ volume.

using this model (Serna and Martin 2006; Szymanski et al. 2000), many of which encode several classes of transcriptional factors including MYB, WD-40, bHLH and HD-ZIP. However, experimental evidence has suggested that this model is limited to plants that belong to the Rosid division of the Eudicots (Serna and Martin 2006). The PALE ALEURONE COLOUR (PAC) gene in maize that encodes a WD repeat protein can complement the glabrous phenotype of ttg1 mutant in Arabidopsis. Interestingly, the pac mutant has no trichome phenotype (Carey et al. 2004). In this study, a WUSCHEL-like homeobox (WOX) gene was identified that controlled trichrome development on the leaf and hull. These results suggested a separate pathway for trichome development in monocot plants and provided new evidence for the convergent evolution of trichome development pathways.

The WOX genes, which are specifically expressed in plants, form a large family that is a subgroup of the homodomain (HD)-containing transcription factors. WOX members characterized to date are involved in regulating diverse aspects of development. LOC_Os11g01130 (Wox3/ OsNS2) is involved in the direct repression of YAB3, which is required for rice leaf development (Dai et al. 2007). OsWOX11 is an auxin- and cytokinin-responsive gene and is required to activate shoot-borne crown root development (Zhao et al. 2009). Ectopic expression of QHB affects normal shoot and leaf development (Kamiya et al. 2003). In this study, a member of the WOX3 subfamily was demonstrated to be involved in trichome development.

In addition to OsNS2, several other WOX3 genes have been investigated. AtWOX3/PRS is expressed in the periphery of the shoot meristem and recruits founder cells from all meristem layers to form lateral domains of both vegetative and floral organs. Ectopic expression of PRS forms multicellular bulges on the stem and sepal (Matsumoto and Okada 2001). The maize duplicate genes narrow sheath 1 (NS1) and narrow sheath 2 (NS2) function in a lateral domain of shoot apical meristems (Nardmann et al. 2004). Mutant leaves miss a large domain, including the leaf margin and mutant internodes are shortened on the marginal side of the stem (Nardmann et al.2007). NS and PRS show conserved function during the recruitment of organ founder cells from the lateral domain of plant meristems (Nardmann et al. 2004). However, none of these genes are involved in trichome development. The presence of orthologs in monocots, such as $O S W O X 3 B$ in rice, which root outside NS/PRS branching, indicated the occurrence of duplication after the separation of monocot and dicot species (Nardmann et al. 2007). The expression patterns of duplicated no-NS/PRS branching paralogs were different from those of NS/PRS branching paralogs, which indicates subfunctionalization (Nardmann et al. 2007). The new function identified in WOX3 members outside NS/PRS branching in this study also supports the occurrence of subfunctionalization in this gene family.

In this study, the expression of $O s W O X 3 B$ was only detected in the panicle of TN1. It is reported that PRS expresses only at a specific developmental stage (Matsumoto and Okada 2001). The negative detection of OsWOX3B in leaf in this study could also be due to the spatiotemporal expression of this gene. Interestingly, OsWOX3B expression was detected in the rice panicle of TN1 but not HMK rice. The transgenic OsWOX3B RNAi lines displayed a glabrous phenotype. These results suggested that OsWOX3B expression was suppressed in Yunnan Nuda Rice. However, no 
differences were identified in the coding or promoter sequences of this gene in comparisons of the HMK and pubescent Nipponbare varieties. It can be speculated that the glabrous phenotype of Yunnan Nuda rice results from epigenetic variations, such as DNA methylation.

Glabrous germplasms have been used to develop dust pollution free rice varieties. In this study, no differences were identified in the $O s W O X 3 B$ gene sequences of pubescent rice and glabrous Yunnan Nuda rice, which would provide an advantageous functional molecular marker for breeding purposes. However, a dominantly inherited glabrous phenotype is obtained by RNAi suppression of $O s W O X 3 B$, thus indicating a novel future strategy for the development of glabrous rice, particularly glabrous hybrid rice.

\section{Conclusion}

Yunnan Nuda Rice exhibited a typical glabrous phenotype. The Nuda phenotype was shown to be controlled by a pair of recessive genes allelic with the wellcharacterized GL-1 locus. The NUDA/GL-1 locus was fine mapped to a $28.5 \mathrm{~kb}$ region. A WUSCHEL-like homeobox gene $(O s W O X 3 B)$ was confirmed as the candidate gene by RNAi and complementation analyses.

\section{Methods}

\section{Plant materials}

In this study, Yunnan Nuda Rice variety HMK was used to identify the NUDA gene. The American glabrous rice variety with $g l-1$ gene Lemont was used for allelic tests. The pubescent indica variety TN1 was crossed with HMK to construct the mapping population. The pubescent variety Nipponbare was used as the receptor of Os WOX3B RNAi. All plants were grown in paddy fields in Shanghai, Hangzhou China during the summer and Hainan, China during the winter.

\section{Scanning electron microscope analysis}

Leaves were dissected, fixed overnight at $4^{\circ} \mathrm{C}$ in FAA (formalin:glacial acetic:70\% ethanol $=1: 1: 18$ ) and dehydrated in a graded ethanol series (50, 70, 80, 90, 95 and $100 \%)$. Samples were critical-point-dried, mounted, sputter-coated with platinum and observed and photographed using a scanning electron microscope (JSM6360LV; JEOL Ltd, Tokyo).

\section{DNA extraction and molecular marker analysis}

Rice genomic DNA was extracted from fresh leaves according to the CTAB method (Murray and Thompson 1980). SSR markers information was obtained from Gramene (www.gramene.org). To construct a high-density linkage map for fine mapping in the target region, new insertion/deletion (InDel) markers and single nucleotide polymorphism (SNP) markers were developed according to the sequence differences between indica var. 93-11 and japonica var. Nipponbare (http://www.ncbi.nlm.nih.gov). Primers flanking the InDel and SNP polymorphisms were designed using the Primer Premier 5.0 program and tested on the parent varieties.

\section{Linkage map and gene mapping}

Two newly developed markers and two polymorphic SSR markers (RM17713 and RM13) were used to construct a linkage map of NUDA/GL-1 using Mapmaker/ Exp 3.0 (Lander et al 1987) on the basis of the 158plant $\mathrm{F}_{2}$ population. The marker order and the genetic distance between every two adjacent markers were determined in the target region on chromosome 5 . These informative molecular markers and newly developed markers were used for genotyping each plant of the $F_{2}$ population to identify recombinants in the target region. The linkage relationship between markers and the NUDA/GL-1 locus was analyzed. By assaying the recombinant events, the $N U D A / G L-1$ locus was narrowed down to a $28.5 \mathrm{~kb}$ region on the AP001 111 PAC clone.

\section{RNA isolation, RT-PCR analysis and RACE}

Total RNA was extracted from various rice tissues using EASYspin (Yuanpinghao, Tianjin). The extracted RNA was treated with RNase-free DNaseI (Frementas) to eliminate genomic DNA contamination according to the protocols recommended by the manufacturer.

First strand cDNA was synthesized from $2.5 \mu \mathrm{g}$ total RNA using the RevertAid ${ }^{\mathrm{TM}}$ First Strand cDNA Synthesis Kit (Fermentas, K1622). RT-PCR was performed with pairs of locus specific primers as follows:

LOC_Os05g02730, forward: 5'-

CTTACACCACCAGCTACTACTACCC-3', reverse, 5'CTAGACGACGTCATGCTGCTCT-3';

LOC_Os05g02740, forward: 5’-

TGGGTTTCCTGCAAAACACT-3', reverse 5'

CTACCGCCAGGCTTCTTGTA-3';

LOC_Os05g02750/ LOC_Os05g02754, forward: 5'-

TGTTCATCACCACGATCTGC-3', reverse 5'-

TGAAGAAGCTGAGGGAGGA-3')

Rice OsActin1 control gene, forward: 5'-

TGCTATGTACGTCGCCATCCAG-3', reverse, 5'AATGAGTAACCACGCTCCGTCA-3').

To obtain the full length-cDNA of LOC_Os05g02730, 5' and 3' RACE were undertaken by using 5'RACE System for Rapid Amplification of cDNA Ends Version 2.0 (Invitrogen, Catalog no.18374-058) and 3'-Full RACE Core Set Ver.2.0 (TaKaRa, Code: D314). The GSP1 primer for 5' RACE is: 5'-CTAGACGACGTCA 
TGCTGCTCT-3'. The GSP1 primer for 3' RACE is:5'CTTACACCACCAGCTACTACTACCC-3'.

\section{In silico analysis of NUDA/GL-1/OsWOX3B protein}

Conserved domain in NUDA/GL-1/OsWOX3B protein was searched in NCBI (http://www.ncbi.nlm.nih.gov/ Structure/cdd/wrpsb.cgi). Blast search parameters were set as the default.

To search the WOX genes in Arabidopsis and rice, BLAST was performed using the Blastp program to search the NCBI Non-redundant Protein Sequences Database (http://blast.ncbi.nlm.nih.gov/) using the homeodomain sequence of AtWUS as a BLAST query. The collected amino acid sequences were aligned using the CLUSTALW program (http://www.ddbj.nig.ac.jp/ search/clustalw-j.html) with standard parameters. A phylogenetic tree was generated with the neighborjoining method and 1,000 bootstrap samples were generated to assess support for the inferred relationships.

\section{Vector construction and transformation}

LOC_Os05g02730 was amplified with its $2 \mathrm{~kb}$ native promoter from Nipponbare genome DNA using primers 02730-clone-f (5'-GGTGTAACGTCTGCCCAAGT-3') and 02730-clone-r (5'-CATTCCATCCATACGCTTGA3 '). The $4.2 \mathrm{~kb}$ PCR product was cloned into the pMD18T (TaKaRa) vector and sequenced. The verified clone was digested with HindIII and EcoRI and the DNA fragment was cloned into pCAMBIA1301 (www.cambia. org). To construct the LOC_Os05g02730 RNAi vector, the third exon was amplified using primer 02730-RNAi-f (5'-GGGGACAAGTTTGTACAAAAAAGCAGGCTTC GGAGGCGGCGGCGTGGTGT-3') and 02730-RNAi-r (5'-GGGGACCACTTTGTACAAGAAAGCTGGGTCC TAGACGACGTCATGCTGCT-3') and the fragment was cloned into the gateway RNAi vector pANDA35HK (Miki et al. 2005) (http://www.invitrogen.com/site/us/ en/home/Products-and-Services/Applications/Cloning/ Gateway-Cloning/GatewayC-Misc/Protocols.html\#bp). The complementary and RNAi vectors were introduced into HMK and Nipponbare, respectively, by Agrobacterium tumefaciens-mediated transformation under selection with hygromycin at $50 \mathrm{mg} / \mathrm{l}$ (Toki 1997). The RNAi lines were verified with primers Guslinker-f (5'-CATGAAGATGCG GACTTACG-3') and Guslinker-r (5'-ATCCACGCCGTA TTCGG-3').

\section{Agronomic traits and evaluation of efficiency of grain packaging}

The agronomic traits of the Nipponbare and the homozygous T2 generation of three independent OsWOX3/ NUDA/GL-1 RNAi transgenic lines were individually surveyed during the summer of 2011 in Songiiang, Shanghai. Factors that included plant height, tillering number, grains per panicle, seed setting rate and 1,000grain weight $(\mathrm{g})$ were measured. The packaging efficiencies of the Nipponbare and the three RNAi transgenic lines were evaluated by measuring the number and weight of dried grains (about 10\% of seed moisture contents) in $100 \mathrm{ml}$ volume. Grain was harvested from four plants of each line. Mean values were compared and analyzed using a t-test.

\section{Additional files}

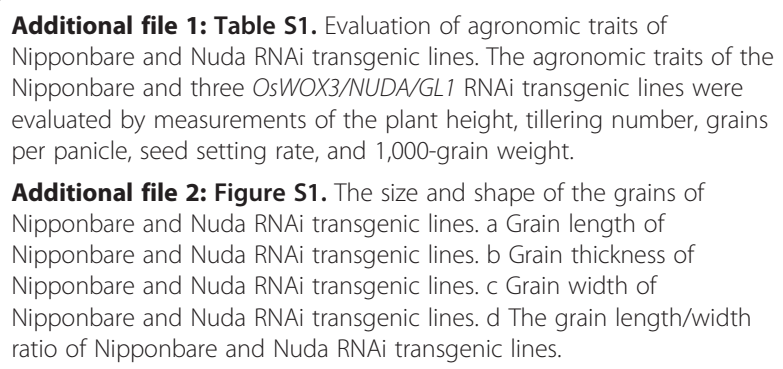

Additional file 2: Figure S1. The size and shape of the grains of Nipponbare and Nuda RNAi transgenic lines. a Grain length of Nipponbare and Nuda RNAi transgenic lines. b Grain thickness of Nipponbare and Nuda RNAi transgenic lines. c Grain width of Nipponbare and Nuda RNAi transgenic lines. d The grain length/width ratio of Nipponbare and Nuda RNAi transgenic lines.

\section{Competing interests}

The authors declare that they have no competing interests.

\section{Authors' contributions}

$\mathrm{HZ}$ and $\mathrm{KW}$ performed the characterization of Yunnan nuda rice, mapping, cloning and analyzing the NUDA/GL-1/OSWOX3B gene. YW and YP performed the rice transformation. $\mathrm{FH}$ and $\mathrm{LW}$ performed the investigation of the agronomic traits. $\mathrm{BH}$ and $\mathrm{QQ}$ conceived the proposal, corrected the drafted manuscript. ST generated financial support for the study and corrected the final manuscript. All authors read and approved the final manuscript.

\section{Acknowledgments}

This research was supported by grants from the National Basic Research Program of China (2009CB1 19000), the Ministry of Agriculture of China for Transgenic Research (2011ZX08009-003, 2011ZX08001-005), the National Science Foundation of China (31000094, 30970246, 31100188, 31161130533), and the Natural Science Foundation of Jiangsu Province, China (BK2010294).

\section{Author details}

${ }^{1}$ Shanghai Institute of Plant Physiology and Ecology, Shanghai Institute for Biological Sciences, The Chinese Academy of Sciences, Shanghai 200032, China. ${ }^{2}$ State Key Laboratory of Rice Biology, China National Rice Research Institute, Chinese Academy of Agricultural Sciences, Hangzhou 310006, China. ${ }^{3}$ Institute of Food Crops, Yunnan Academy of Agricultural Sciences, Kunming 650205, China. ${ }^{4}$ Puer Agricultural Research Institute, Puer 66500, China.

Received: 30 March 2012 Accepted: 27 September 2012 Published: 3 October 2012

\section{References}

Carey CC, Strahle JT, Selinger DA, Chandler VL (2004) Mutations in the pale aleurone color1 regulatory gene of the Zea mays anthocyanin pathway have distinct phenotypes relative to the functionally similar TRANSPARENT TESTA GLABRA1 gene in Arabidopsis thaliana. Plant Cell 16:450-464

Dai M, Hu Y, Zhao Y, Liu H, Zhou DX (2007) A WUSCHEL-LIKE HOMEOBOX gene Represses a YABBY gene expression required for rice leaf development. Plant Physiol 144:380-390

Foster KW, Rutger JN (1978) Independent segregation of semi-dwarfing genes and a gene for pubescence in rice. J Hered 69:137-138

Gibson PT, Maiti RK (1983) Trichomes in segregating generations of Sorghum matings.1. Inheritance of presence and density. Crop Sci 23:73-75

Haecker A, Gross-Hardt R, Geiges B, Sarkar A, Breuninger H, Herrmann M, Laux T (2004) Expression dynamics of WOX genes mark cell fate decisions during 
early embryonic patterning in Arabidopsis thaliana. Development 131:657-668

Hulskamp M, Schnittger A (1998) Spatial regulation of trichome formation in Arabidopsis thaliana. Semin Cell Dev Biol 9:213-220

Hulskamp M (2004) Plant trichomes: a model for cell differentiation. Nat Rev Mol Cell Biol 5:471-480

Johnson CS, Kolevski B, Smyth DR (2002) TRANSPARENT TESTA GLABRA2, a trichome and seed coat development gene of Arabidopsis, encodes a WRKY transcription factor. Plant Cell 14:1359-1375

Kamiya N, Nagasaki H, Morikami A, Sato Y, Matsuoka M (2003) Isolation and characterization of a rice WUSCHEL-type homeobox gene that is specifically expressed in the central cells of a quiescent center in the root apical meristem. Plant J 35:429-441

Karkkainen K, Agren J (2002) Genetic basis of trichome production in Arabidopsis lyrata. Hereditas 136:219-226

Kirik V, Simon M, Wester K, Schiefelbein J, Hulskamp M (2004) ENHANCER of TRY and CPC 2 (ETC2) reveals redundancy in the region-specific control of trichome development of Arabidopsis. Plant Mol Biol 55:389-398

Kumar KA, Andrews DJ (1993) Genetics of qualitative traits in pearl-millet - a Review. Crop Sci 33:1-20

Lander ES, Green P, Abrahanson J, Barlow A, Daly MJ, Lincon SE, Newburg L (1987) MAPMAKER: an interactive computing package for constructing primary genetic linkages of experimental and natural populations. Genomics 1:174-181

Larkin JC, Oppenheimer DG, Pollock S, Marks MD (1993) Arabidopsis GLABROUS1 gene requires downstream sequences for function. Plant Cell 5:1739-1748

Leisle D (1974) Genetics of leaf pubescence in wheat. Crop Sci 14:173-174

Li W, Wu J, Weng S, Zhang D, Zhang Y, Shi C (2010) Characterization and fine mapping of the glabrous leaf and hull mutants ( $\mathrm{g} \mid 1)$ in rice (Oryza sativa L.). Plant Cell Rep 29:617-627

Lim HH, Domala Z, Joginder S, Lee SH, Lim CS, Abu Bakar CM (1984) Rice millers' syndrome: a preliminary report. Br J Ind Med 41:445-449

Lloyd AM, Payne CT, Zhang F (2000) GL3 encodes a bHLH protein that regulates trichome development in arabidopsis through interaction with GL1 and TTG1. Genetics 156:1349-1362

Ma JF, Huang CF, Yamaji N, Mitani N, Yano M, Nagamura Y (2009) A bacterialtype $A B C$ transporter is involved in aluminum tolerance in rice. Plant Cell 21:655-667

Marks MD (1997) Molecular genetic analysis of trichome development in Arabidopsis. Annu Rev Plant Physiol Plant Mol Biol 48:137-163

Matsumoto N, Okada K (2001) A homeobox gene, PRESSED FLOWER, regulates lateral axis-dependent development of Arabidopsis flowers. Genes Dev 15:3355-3364

Miki D, Itoh R, Shimamoto K (2005) RNA silencing of single and multiple members in a gene family of rice. Plant Physiol 138:1903-1913

Moose SP, Lauter N, Carlson SR (2004) The maize macrohairless1 locus specifically promotes leaf blade macrohair initiation and responds to factors regulating leaf identity. Genetics 166:1451-1461

Murray MG, Thompson WF (1980) Rapid isolation of high molecular weight plant DNA. Nucleic Acids Res 8:4321-4325

Nagao S, Takahashi M, Kioshita T (1960) Genetical studies on rice plant, XXV: Inheritance of three morphological characters, pubescence of leaves and floral glumes, and deformation of empty glumes. Journal of the Faculty of Agriculture, Hokkaido University 51:299-314

Nardmann J, Ji J, Werr W, Scanlon MJ (2004) The maize duplicate genes narrow sheath1 and narrow sheath2 encode a conserved homeobox gene function in a lateral domain of shoot apical meristems. Development 131:2827-2839

Nardmann J, Zimmermann R, Durantini D, Kranz E, Werr W (2007) WOX gene phylogeny in Poaceae: a comparative approach addressing leaf and embryo development. Mol Biol Evol 24:2474-2484

Oppenheimer DG, Herman PL, Sivakumaran S, Esch J, Marks MD (1991) A myb gene required for leaf trichome differentiation in Arabidopsis is expressed in stipules. Cell 67:483-493

Payne CT, Zhang F, Lloyd AM (2000) GL3 encodes a bHLH protein that regulates trichome development in arabidopsis through interaction with GL1 and TTG1. Genetics 156:1349-1362

Qin F, Zhang D, Lin X, Xie Y (1997) Classification of Yunnan Nuda compatible varieties. J Huazhong Agricultural University 16:320-324

Rutger JN, Mackill DJ (2001) Application of Mendelian genetics in rice breeding. In: Rice Genetics IV, Proceedings of the Fourth International Rice Genetics Symposium, 22-27 October 2000, Los Baños, Philippines. Science Publishers,
Inc and Los Baños (Philippines): International Rice Research Institute, Enfield, $\mathrm{NH}$ (USA), p 29

Sarkarung S, Collins F (1977) Inheritance of leaf pubescence in oats. AgronAbstr $77: 7$

Sato K, Takeda K (1992) Genetic analysis of large trichomes on the barley leaf blade. Barley Genet Newsl 22:50-52

Scanlon MJ, Schneeberger RG, Freeling M (1996) The maize mutant narrow sheath fails to establish leaf margin identity in a meristematic domain. Development 122:1683-1691

Schellmann S, Schnittger A, Kirik V, Wada T, Okada K, Beermann A, Thumfahrt J, Jurgens G, Hulskamp M (2002) TRIPTYCHON and CAPRICE mediate lateral inhibition during trichome and root hair patterning in Arabidopsis. EMBO J 21:5036-5046

Serna L, Martin C (2006) Trichomes: different regulatory networks lead to convergent structures. Trends Plant Sci 11:274-280

Southwood R (1986) Plant surfaces and insects - an overview. In: Juniper B, Southwood R (eds) Insects and the plant surface. Edward Arnold (Publishers) Ltd, London, Sydney \& Baltimore, pp 1-22

Szymanski DB, Lloyd AM, Marks MD (2000) Progress in the molecular genetic analysis of trichome initiation and morphogenesis in Arabidopsis. Trends Plant Sci 5:214-219

Toki S (1997) Rapid and efficient Agrobacterium-mediated transformation in rice. Plant Mol Biol Rep 15:16-21

Vandenbussche M, Horstman A, Zethof J, Koes R, Rijpkema AS, Gerats T (2009) Differential recruitment of WOX transcription factors for lateral development and organ fusion in Petunia and Arabidopsis. Plant Cell 21:2269-2283

Walker AR, Davison PA, Bolognesi-Winfield AC, James CM, Srinivasan N, Blundell TL, Eschc JJ, Marksc MD, Graya JC (1999) The TRANSPARENT TESTA GLABRA1 locus, which regulates trichome differentiation and anthocyanin biosynthesis in Arabidopsis, encodes a WD40 repeat protein. Plant Cell 11:1337-1350

Wang D, Sun S, Fa G, Lu X, Li Z, Ren G (2009) Mapping a rice glabrous gene using simple sequence repeat markers. Rice Sci 16:93-98

Werker E (2000) Trichome diversity and development. Adv Bot Res 31:1-35

Yu ZH, Mccouch SR, Kinoshita T, Sato S, Tanksley SD (1995) Association of morphological and RFLP markers in rice (Oryza-Sativa L). Genome 38:566-574

Zhang X, Zong J, Liu J, Yin J, Zhang D (2010) Genome-wide analysis of WOX gene family in rice, sorghum, maize, Arabidopsis and poplar. J Integr Plant Biol 52:1016-1026

Zhao Y, Hu Y, Dai M, Huang L, Zhou DX (2009) The WUSCHEL-related homeobox gene WOX11 is required to activate shoot-borne crown root development in rice. Plant Cell 21:736-748

doi:10.1186/1939-8433-5-30

Cite this article as: Zhang et al: A WUSCHEL-like homeobox gene, OsWOX3B responses to NUDA/GL-1 locus in rice. Rice 2012 5:30.

\section{Submit your manuscript to a SpringerOpen ${ }^{\odot}$ journal and benefit from:}

- Convenient online submission

- Rigorous peer review

- Immediate publication on acceptance

- Open access: articles freely available online

- High visibility within the field

- Retaining the copyright to your article

Submit your next manuscript at $>$ springeropen.com 